\title{
Vitamin A status among children in China
}

\author{
Jiang Jingxiong ${ }^{1, *}$, André Michael Toschke ${ }^{2}$, Rüdiger von Kries² ${ }^{2}$, Berthold Koletzko ${ }^{3}$ \\ and Lin Liangming ${ }^{4}$ \\ ${ }^{1}$ Department of Child Health Care, National Center for Women's and Children's Health, Chinese CDC, No. 13 Dong \\ Tu Cheng Road, Chaoyang District, Beijing 100013, China: ${ }^{2}$ Institute for Social Pediatrics and Adolescent Medicine, \\ Division of Epidemiology, Ludwig-Maximilians University, Munich, Germany: ${ }^{3} \operatorname{Dr}$ von Hauner Children's Hospital, \\ University of Munich, Germany: ${ }^{4}$ Capital Institute of Pediatrics, Beijing, China
}

Submitted 1 September 2005: Accepted 10 January 2006

\begin{abstract}
Objective: The objective of this study was to assess the prevalence of vitamin A deficiency (VAD) in children under 6 years old in China and to identify risk groups for VAD.

Design: A cross-sectional survey was conducted in 14 provinces from coastal, inland and western geographic areas in China.

Setting: One city (urban) and two counties (rural) were randomly selected from each province as survey areas.

Subjects: About 200 children aged 0-6 years were randomly selected in each survey area. A blood sample was collected from each child. Data on sociodemographics and nutrition were obtained by interview of the mother or principal caregiver. Fluorescence microanalysis was used to analyse serum retinol concentration. Results: VAD (serum retinol $<0.7 \mu \mathrm{moll}^{-1}$ ) was observed in 957 out of 7826 children aged $0-6$ years (12.2\% of the entire study population), whereas severe VAD (serum retinol $<0.35 \mu \mathrm{moll}^{-1}$ ) was found in 39 children $(0.5 \%)$. The highest prevalences of $\mathrm{VAD}$ at $>1$ year of age were observed among children of mothers with minority ethnicity (22.7\%) or poor education (19.8\%) and in the poor western area (17.4\%). Conclusions: VAD is a nutritional problem in children in China. Children living in the poor western area, having a mother with minority ethnicity or a mother with poor education have a high risk of VAD.
\end{abstract}

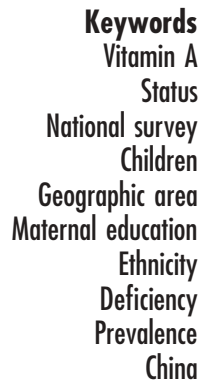

Vitamin A deficiency (VAD) is a major micronutrient problem in many developing countries. There are estimates that worldwide approximately 140 million children of pre-school age suffer from VAD every year and of these 4.4 million suffer from xerophthalmia ${ }^{1,2}$. Nearly half of all VAD occurs in South and Southeast Asia ${ }^{3}$. VAD might increase children's susceptibility to infection, reduce physical growth and decrease the possibility of survival from serious illness. A number of epidemiological studies have identified an association between VAD and higher rates of mortality ${ }^{4,5}$.

VAD in African countries is associated with insufficiently varied diets, poor maternal education and low economic status $^{6,7}$. Young children are susceptible to VAD if their dietary intake of the essential vitamin is inadequate. Although the prevalence of protein-energy deficiency malnutrition has decreased in most Chinese areas with economic development in recent years ${ }^{8,9}$, micronutrient deficiency might still be an issue. A study from western China showed that VAD was a health problem among children that had affected their growth ${ }^{10}$. At the 1990 World Summit for Children, the World Health Organization
(WHO), the United Nations Children's Fund and governments of the world committed themselves to eliminate VAD as a public health problem by the year 2000. Elimination of VAD was declared a National Programme of Action for Child Health in China in the 1990s. In order to obtain baseline information on vitamin A status and identify an intervention strategy, the first cross-sectional survey of VAD was conducted from November 1999 to July 2000 in the whole of China. The present paper estimates vitamin A status and the prevalence of VAD, and analyses its relationship to sociodemographic and dietary factors.

\section{Methods}

\section{Study population}

Data from 8681 children aged 0-6 years were collected. The sampling design consisted of a three-step stratified random sampling. In the first step, four or five provinces were selected at random in each of the three geographic areas defined by the State Statistics Bureau of China ${ }^{11}$ coastal, inland and west. In the second step, within each province one city and two counties were selected at 
random. In each city, two districts and within the districts three communities were selected at random. In rural areas three townships and within townships two villages were selected at random (Fig. 1). In each community or village, 11 or 12 children in each age group ( $0-23$ months, 24-47 months and 48-71 months) were randomly selected. Overall, less than $2 \%$ of the sample was classified as a nonresponder due to parental refusal to participate in the study or failure to provide a blood sample for analysis.

Since information on vitamin supplements was provided by only $8 \%$ of parents, these children were excluded from the analysis. Additionally we excluded children having a disease in the two weeks immediately before the examination. Thus the analysis was confined to 7826 healthy children with complete information and plausible information on explanatory variables and vitamin A level. Furthermore, in the first year of life, a large amount of heterogeneity is likely due to variable initiation and duration of breast-feeding and differences in the onset of introducing solids. We therefore confined the multivariate analyses to a sub-sample of 6885 children aged 1-6 years. The proportion of children from cities versus children from rural areas was 1:3 (Fig. 1). This survey was approved by the Ministry of Health of China and parental consent was obtained for all children before enrolment.

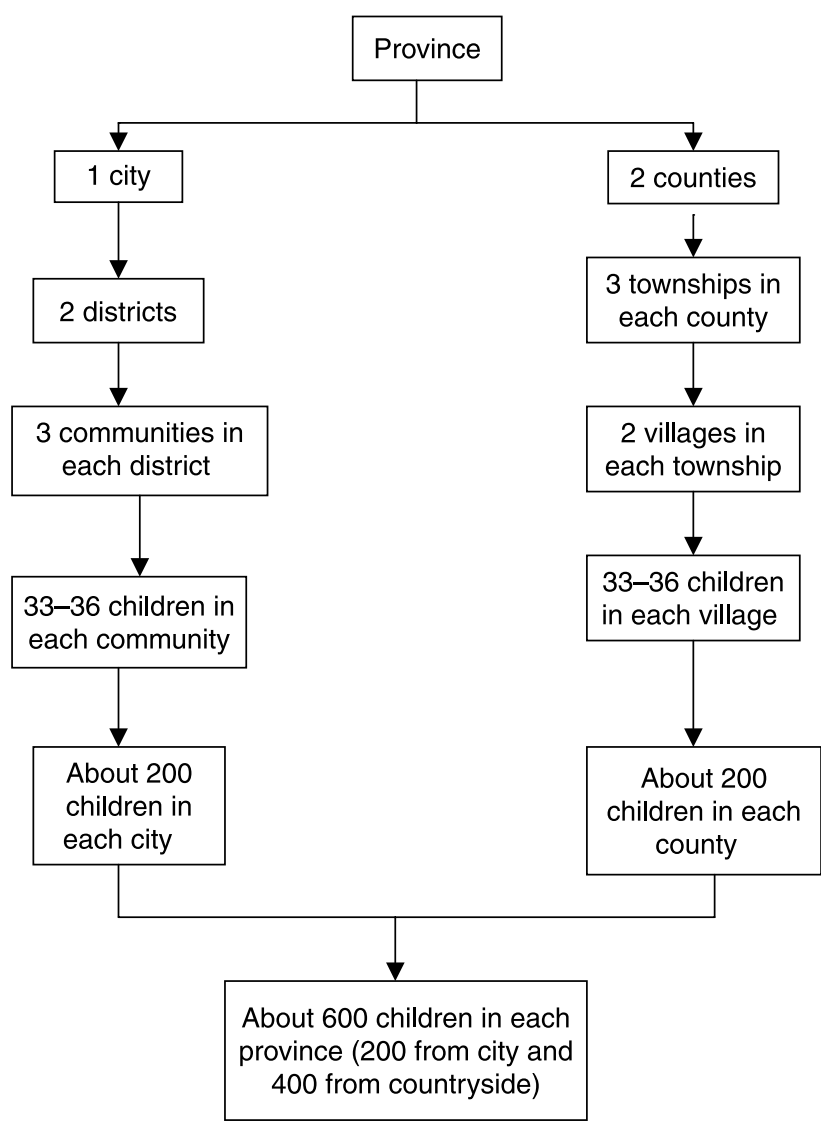

Fig. 1 Sampling design

\section{Measurements}

A $200 \mu \mathrm{l}$ capillary blood sample was taken from each child by puncture of the left ring finger. The blood samples were protected from light, stored in cool boxes and centrifuged for $10 \mathrm{~min}$ within $4-6 \mathrm{~h}$ after collection. The serum was protected from light and stored at $-20^{\circ} \mathrm{C}$ for up to 9 days and then transported for a maximum of $6 \mathrm{~h}$ in a cool box before it was stored at $-70^{\circ} \mathrm{C}$ in the laboratory of the Capital Institute of Pediatrics in Beijing, where the serum samples were analysed within 3 months.

The serum samples were analysed for retinol using fluorescence microanalysis under a strict quality control protocol. A consultant was sent from the Centers for Disease Control and Prevention (CDC), Atlanta, GA, USA to direct the serum retinol measurements at the Capital Institute of Pediatrics, Beijing. The fluorescence spectrophotometer was an RF-540 from Shimadzu (Kyoto, Japan). Standard serum samples $(n=100)$ from CDC, Atlanta were analysed for serum retinol as a quality control measure. The concentration of retinol in the standard serum samples, as measured by high-performance liquid chromatography assay (HPLC), was $1.336 \mu \mathrm{moll}^{-1}$. The acceptable range of measurement for the serum retinol concentration was $1.225-1.575 \mu \mathrm{moll}^{-1}$. The range of serum retinol concentration for the 100 serum samples measured by fluorescence microanalysis was 1.278$1.545 \mu \mathrm{moll}^{-1}$. The coefficient of variation for repeat measurements was $2.9-9.9 \%$.

Serum retinol concentration of $<0.7 \mu \mathrm{moll}^{-1}$ was used as a cut-off point for VAD and serum retinol concentration $<0.35 \mu \mathrm{moll}^{-1}$ as the cut-off point for severe VAD, in accordance with widely used international definitions ${ }^{2,12}$ and the definition recommended by the Chinese Association of Pediatrics ${ }^{13}$. Serum retinol concentration $<1.05 \mu \mathrm{mol}^{-1}$ was defined as marginal $\mathrm{VAD}^{3}$.

\section{Interview}

The interview on diet was based on the Helen Keller International Food Frequency Method to obtain information on the frequency of consumption of dietary sources of vitamin A intake of the children. The mothers or caregivers were asked how often their children had milk, liver, meats (all kinds of meats excluding liver), fish, eggs and dark green (orange) fresh vegetables in the past week. The Helen Keller Food Frequency Method has been validated to provide an accurate community-level measure for identification of a diet associated with VAD and has been used elsewhere ${ }^{14,15}$. Additionally, information on parental education and ethnicity was obtained within the interview. Education was categorised into four levels: primary school not completed (incomplete or no primary school), primary school (graduated from primary school), high school (graduated from high school or incomplete high school) and college or above (graduated from college or above or incomplete college). Ethnicity was categorised as Han (majority) and non-Han (minority ethnicity). 
The questions were pre-tested; after the initial training course, each of 28 participating physicians interviewed 10 individuals for pre-testing. The questions were then modified to increase practicability, reliability and validity. The interviews were performed by two trained local doctors and one person from the study team at each field site. In all households, the primary respondent was the mother of the selected child or the principal caregiver.

\section{Statistical methods}

The prevalence of VAD was estimated with 95\% exact confidence limits based on the binomial distribution ${ }^{16}$. A multiple linear regression model was used to assess the impact of potential predictors on serum retinol concentrations $\left(\mu \mathrm{mol} \mathrm{l}^{-1}\right)$. Additionally a logistic regression model was estimated with VAD defined at a serum retinol concentration of $<0.7 \mu \mathrm{moll}^{-1}$. All covariates associated with serum retinol concentrations or VAD were considered for a multiple logistic or a linear regression model, respectively. Only significant $(P<0.05)$ predictors were included in the final models.

Outliers (data points which do not appear to follow the characteristic distribution of the rest of the data) were excluded from the analysis to minimise the impact of measurement errors or other anomalies. For each individual outcome measurement we tested whether its exclusion changed the standard deviation of the outcome variable by more than $10 \%$. Any observation fulfilling this criterion was excluded as an outlier. Regression diagnostics further included examination of residuals, nonconstant error of variance (heteroscedasticity) and non-linearity. Multicollinearity of respective covariates was identified ${ }^{17}$ by a variance inflation factor $>10$.

All calculations were carried out with the software package SAS version 8.2 (SAS Institute Inc., Cary, NC, USA).

\section{Results}

VAD (serum retinol $<0.7 \mu \mathrm{moll}^{-1}$ ) was observed in 957 out of 7826 children (12.2\% of the entire study population), whereas severe VAD (serum retinol $<0.35 \mu \mathrm{mol}^{-1}$ ) was observed in $39(0.5 \%)$. Serum retinol concentrations were marginal $\left(0.7\right.$ to $\left.<1.05 \mu \mathrm{moll}^{-1}\right)$ in 3140 (40.1\%) children aged $0-6$ years old.

The highest prevalence of VAD (serum retinol $<0.7 \mu \mathrm{moll}^{-1}$ ) was observed in children of mothers without Han ethnicity (22.7\%) or lower maternal education (primary school not completed) (19.8\%) and in children living in the poor western area (17.4\%) (Table 1). The prevalence of VAD showed an inverse relationship to children's age, level of maternal education and region, with an increase in the order of coastal area followed by the inland area and the western area (Table 1).

The left-shifted lower percentiles for serum retinol concentration in the various strata were not due to skewed distributions of the serum retinol levels. Rather the explanatory variables were related to shifts of the entire distribution of the serum retinol levels. As an example, the association between Han ethnicity and serum retinol concentration is shown in Fig. 2. In children of Han mothers, the crude mean serum retinol concentration was $0.16 \mu \mathrm{moll}^{-1}$ higher than in children of non-Han mothers.

Table 1 Prevalence of VAD (serum retinol $<0.7 \mu \mathrm{molI}^{-1}$ ) among Chinese children

\begin{tabular}{lc}
\hline Explanatory variable & $\begin{array}{c}\text { Prevalence }(\%) \\
\text { of VAD }(95 \% \mathrm{Cl})\end{array}$ \\
\hline Entire population $(n=7826)$ & $12.2(11.5-13.0)$ \\
Age (years) & \\
$0(n=941)$ & $26.6(23.8-29.5)$ \\
$1(n=1331)$ & $14.1(12.2-16.0)$ \\
$2(n=1341)$ & $11.3(9.7-13.2)$ \\
$3(n=1334)$ & $10.1(8.6-11.7)$ \\
$4(n=1451)$ & $7.9(6.6-9.4)$ \\
$5(n=1314)$ & $8.4(6.9-10.0)$ \\
$6(n=114)$ & $7.0(3.1-13.4)$ \\
Geographic area* & $17.4(16.0-18.9)$ \\
Western area (low socio-economic status; $n=2799)$ & $11.8(10.7-13.1)$ \\
Inland area (moderate socio-economic status; $n=2822)$ & $6.2(5.2-7.3)$ \\
Coastal area (high socio-economic status; $n=2205)$ & \\
Maternal ethnicity* & $22.7(20.8-24.8)$ \\
Non-Han $(n=1764)$ & $9.2(8.6-9.9)$ \\
Han $(n=6062)$ & \\
Maternal educational level ${ }^{*}$ & $19.8(16.8-23.0)$ \\
Primary school not completed $(n=657)$ & $17.5(16.0-19.2)$ \\
Primary $(n=2248)$ & $9.6(8.8-10.6)$ \\
Middle school $(n=4307)$ & $2.9(1.8-4.6)$ \\
College $(n=614)$ & \\
\hline
\end{tabular}

VAD - vitamin A deficiency; $\mathrm{Cl}$ - confidence interval.

${ }^{*}$ Cochran-Armitage trend test: $P<0.0001$. 


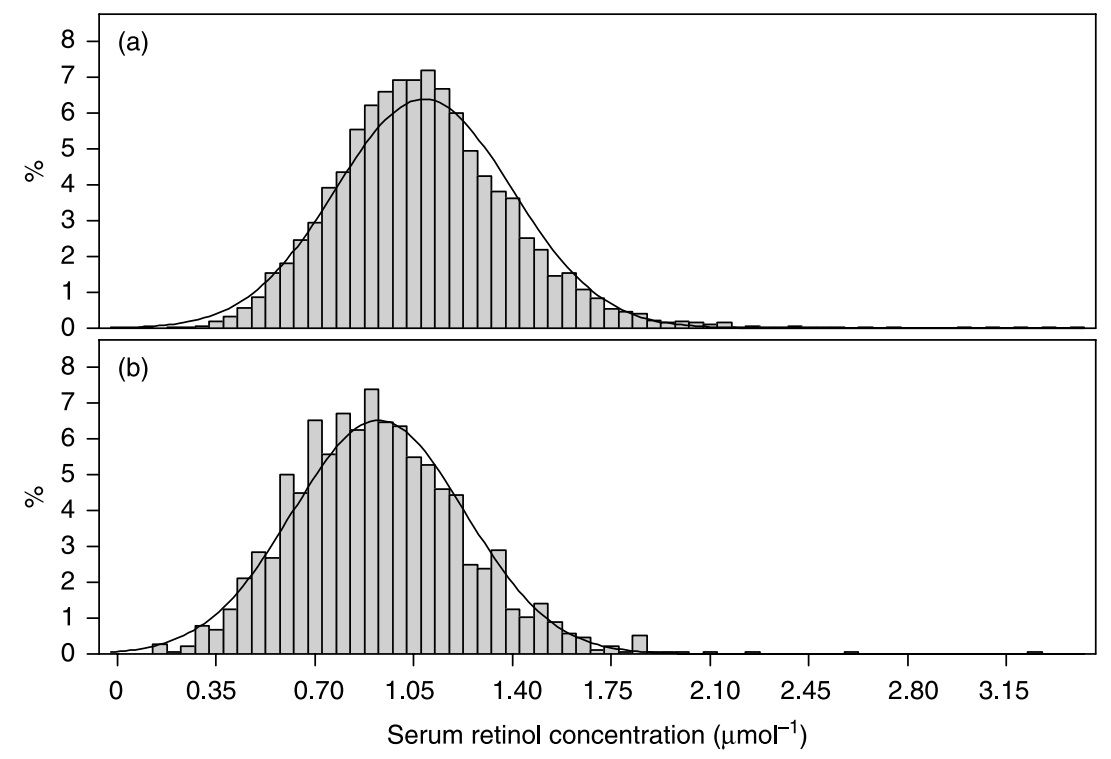

Fig. 2 Effect of mothers' ethnicity on serum retinol concentration among Chinese children aged 1-6 years: (a) Han ethnicity ( $n=6062$; serum retinol concentration $1.09 \pm 0.31 \mu \mathrm{moll}^{-1}$, mean \pm standard deviation, SD); (b) non-Han ethnicity $(n=1764$; serum retinol concentration $0.93 \pm 0.31 \mu \mathrm{mol} \mathrm{I}^{-1}$, mean $\pm \mathrm{SD}$ ). Unadjusted mean difference: $-0.16 \mu \mathrm{mol} \mathrm{I}^{-1}$

In order to assess the mutually independent relationships between the explanatory variables depicted in Table 1 and nutritional intake, the mutually adjusted increment in serum retinol related to each of these explanatory variables is shown in Table 2 for children aged 1 year and older. The child's age, coastal geographic area, high maternal education and Han ethnicity were independently associated with higher vitamin A levels for children.

Similar results were observed for VAD as categorical outcome variable (multiple logistic regression analysis): independent protective effects of Han nationality, high maternal education, coastal area and additional year of life were observed which could not be explained by the consumption of milk, meat or eggs (Table 2). The adjusted odds ratio of belonging to a minority, having a mother with poor education and living in the poor western area, compared with belonging to Han nationality, having a highly educated mother and living in coastal parts of the country (reference), for example, was 11.6 $(11.6=1 / \exp [\ln (0.54)+\ln (0.30)+\ln (0.53)] ;$ see Table $2)$. This was due to a lowered average serum retinol level

Table 2 Significant increases $(P<0.05)$ in mean retinol concentration (multiple linear regression) and significant $(P<0.05)$ adjusted OR for VAD (serum retinol $<0.7 \mu \mathrm{moll}^{-1}$; multiple logistic regression) among $n=6885$ children aged $1-6$ years

\begin{tabular}{|c|c|c|}
\hline Explanatory variable & $\begin{array}{l}\text { Adjusted* increase } \\
\text { in mean retinol } \\
\text { level }\left(\mu \mathrm{moll}^{-1}\right) \text { (SD) }\end{array}$ & $\begin{array}{l}\text { Adjusted† OR for } \\
\operatorname{VAD}(95 \% \mathrm{Cl})\end{array}$ \\
\hline \multicolumn{3}{|c|}{ Geographic area (western area (low socio-economic status) as reference) } \\
\hline Inland area (moderate socio-economic status) & $0.02(0.01)$ & $0.75(0.61-0.91)$ \\
\hline Coastal area (high socio-economic status) & $0.05(0.01)$ & $0.53(0.41-0.69)$ \\
\hline \multicolumn{3}{|l|}{ Maternal ethnicity (non-Han as reference) } \\
\hline Han & $0.08(0.01)$ & $0.54(0.45-0.65)$ \\
\hline \multicolumn{3}{|c|}{ Maternal educational degree (primary school not completed as reference) } \\
\hline Primary & $0.01(0.01)$ & $1.03(0.80-1.34)$ \\
\hline High school & $0.08(0.02)$ & $0.75(0.57-0.99)$ \\
\hline College & $0.16(0.02)$ & $0.30(0.46-0.56)$ \\
\hline \multicolumn{3}{|l|}{ Consumption (within the week prior to examination) } \\
\hline Liver (ever) & $0.03(0.01)$ & - \\
\hline Milk (ever) & $0.08(0.01)$ & $0.57(0.47-0.69)$ \\
\hline Meat (daily) & $0.02(0.01)$ & $0.78(0.65-0.92)$ \\
\hline Fish (ever) & $0.02(0.01)$ & - \\
\hline Eggs (daily) & $0.03(0.01)$ & $0.69(0.56-0.84)$ \\
\hline Per additional year of child's age & $0.02(<0.01)$ & $0.86(0.81-0.91)$ \\
\hline
\end{tabular}

OR - odds ratio; VAD - vitamin A deficiency; SD - standard deviation; $\mathrm{Cl}$ - confidence interval.

${ }^{*}$ Adjusted for all variables displayed in table.

†Adjusted for geographical area, maternal ethnicity, maternal school education and consumption of milk, eggs and meat. 
of $-0.29 \mu \mathrm{moll}^{-1}(-0.29=-0.08+(-0.16)+(-0.05)$; see Table 2).

\section{Discussion}

The main finding of this study was an average prevalence of VAD of $12.2 \%$, including $0.5 \%$ of severe VAD in Chinese children aged $0-6$ years. While the VAD prevalence in the eastern coastal areas was only 6.2\%, it was almost three times as common in the western parts of China. These figures are close to those found in a previous study with small sample size ${ }^{18}$. In the present survey, belonging to an ethnic minority, having a mother with poor education and living in the poor western area accounted for an adjusted odds ratio of 11.6 for VAD compared with belonging to HAN ethnicity, having a highly educated mother and living in coastal parts of the country.

The strengths of this study are a defined random sampling procedure and the use of a standardised method to measure VAD. The study sites covered half the provinces in China. They are likely to reflect the prevalence of VAD in the whole of China because the distribution of socio-economic classes for the selected provinces was similar to that of the national distribution according to the Classification of Chinese Health and Economic Status ${ }^{19}$.

Vitamin A status can be assessed by ophthalmologic examination, measuring serum retinol concentration and the Modified Relative Dose Response. Serum retinol concentration is usually measured by HPLC. For the sake of practicality in the present large epidemiological study, fluorescence microanalysis was used to measure the serum retinol level. The pre-testing showed similar reliability and validity for fluorescence microanalysis as for HPLC. The cut-off values for VAD (serum retinol $<0.35 \mu \mathrm{moll}^{-1},<0.7 \mu \mathrm{moll}^{-1}$ and 0.7 to $<1.05 \mu \mathrm{moll}^{-1}$ ) were chosen as recommended by $\mathrm{WHO}^{1}$. Severe VAD can be defined as serum retinol $<0.35 \mu \mathrm{moll}^{-1}$ or ophthalmologic symptoms ${ }^{2}$. Serum retinol $<1.05 \mu \mathrm{moll}^{-1}$ was considered indicative of potentially suboptimal vitamin A status $^{2,13}$. It appeared that the prevalences of VAD and potentially suboptimal concentrations of serum retinol were high in children in China, whereas the prevalence of severe or clinically manifest VAD was very low.

$\mathrm{VAD}$ is a common nutritional disorder in many developing countries; e.g. in northern Ethiopia, VAD prevalence is $59.5 \%$ in children aged $6-9$ years ${ }^{7}$ and in Nepal the prevalence is $32.3 \%$ among pre-school children $^{20}$. Compared with these and other developing countries $^{6,21}$, the average prevalence of VAD was lower in China. China is a big country with unbalanced economic development. Although VAD prevalence was only $6.2 \%$ in the rich coastal area, which is comparable to data from the $\mathrm{USA}^{22}$, VAD still remains a public health problem in the poor western area where its prevalence was $17.4 \%$.
The available dietary data could not fully explain different serum retinol levels between the various socioeconomic subgroups. The consumption of all animal foods (liver, milk, meat, fish and eggs) is associated with serum retinol concentration. Usually, animal foods (including vitamin-A-rich animal foods) are more expensive than vegetables in China. The higher consumption of animal foods may be a proxy for a higher income or a better diet. It might reflect the effect of dietary intake on vitamin A level indirectly. Dark green vegetables are a major source of vitamin A but, surprisingly, the consumption of dark green vegetables in the week prior to the examination had no relationship with serum retinol concentration. This might be due to a lack of adequate variation in consumption among this population - $82 \%$ had eaten such vegetables daily. The diet in China may be too different from that of those countries where the Helen Keller questionnaire was validated ${ }^{14}$.

A significant ethnic difference in serum retinol concentration was observed in this study. The prevalence of VAD was $22.7 \%$ in children of non-Han mothers compared with $9.2 \%$ in children of Han mothers. According to WHO criteria, a prevalence of $20 \%$ or above for serum retinol $<0.7 \mathrm{mmol}^{-1}$ is indicative of a VAD problem of public health significance ${ }^{23}$. These WHO criteria were based on surveys of children aged 6-71 months. The age range within this survey was similar.

Most of minority groups live in the western parts of China. Their economic status is below that of the Han majority: the per capita income of non-Han people is $80 \%$ of the per capita income of Han people ${ }^{24}$. Surprisingly we found a strong and significant impact of region on the risk for VAD, even after adjustment for maternal education and minority status. There are plenty of data on the socioeconomic differences between eastern and western areas: per capita income in the western area is only $71 \%$ of the per capita income in eastern urban areas ${ }^{25}$. Unfortunately, we lacked individual data on income in relation to regional living costs. This might account for a bias towards unity due to random misclassification of the exposure.

Younger children had a higher prevalence of VAD than older children. This finding is consistent with other reports $^{25}$. It might also be due to the use of one single cut-off point for all age groups, not taking into account potentially physiologically lower vitamin A levels in younger children. Unfortunately, no international agespecific cut-off points for low serum retinol concentration exist for young children. The inverse relationship between age and low serum retinol concentration might be attributed to long-term effects of vitamin-A-rich foods or supplements. Breast milk is a better source of vitamin A for infants than formula feeding. Breastfeeding and fortified complementary feeding should be improved constantly for infants and young children in order to increase the accumulation of nutrients, including vitamin A. 


\section{Conclusion}

A high prevalence of VAD was observed in western provinces compared with eastern areas in China. Particularly minority children and those with mothers having poor education have been identified as risk groups of public health importance. These children should be targeted for preventive measures.

A strategy of vitamin A supplementation has been put into the National Programme of Action for Child Health in China (2001-2010) following the findings of the present national survey. Additionally, after this study vitamin A and iron folic acid supplementation to pregnant women was implemented in some rural provinces in China. However, there is no official health-care policy generally targeting new mothers at children's vitamin A supplementation. Thus, a comprehensive, long-term national strategy is needed to promote sustained improvement in vitamin A status in China. Vitamin A supplementation should be offered during public vaccination consultation hours, regular consumption of vitamin-A-rich foods should be promoted by nutrition education, and breast-feeding and fortified complementary feeding in infants and young children should be improved.

\section{Acknowledgements}

This survey was carried out with the aid of a nutrition grant (YH101-13) from UNICEF. We thank the mothers and children who participated in this study, Ma Guanfu, Tan Zangwen and Liu Min in the Capital Institute of Pediatrics for their laboratory analyses of serum retinol, Liu Yulin for her advice and the members of our field team. Additionally we want to thank the editorial team and the reviewers for their helpful comments to improve the manuscript.

\section{References}

1 World Health Organization (WHO)/United Nations Children's Fund. Indicators for Assessing Vitamin A Deficiency and Their Application in Monitoring and Evaluating Intervention Programmes. Micronutrient Series. Geneva: WHO, 1996.

2 Sommer A, Davidson FR. Assessment and control of vitamin A deficiency: the Annecy Accords. Journal of Nutrition 2002; 132: 2845 S-50S.

3 Underwood BA. Vitamin A in human nutrition: public health considerations. In: Sporn M, Roberts AB, Goodman PS, eds. The Retinoids: Biology, Chemistry and Medicine. New York: Raven Press, 1994.

4 Schultink W. Use of under-five mortality rate as an indicator for vitamin A deficiency in a population. Journal of Nutrition 2002; 132: 2881S-3S

5 Fawzi WW, Herrera MG, Spiegelman DL. A prospective study of malnutrition in relation to child mortality in the Sudan. American Journal of Clinical Nutrition 1997; 65: 1062-9.

6 Schemann JF, Banou AA, Guindo A, Joret V, Traore L, Malvy D. Prevalence of undernutrition and vitamin A deficiency in the Dogon Region, Mali. Journal of the American College of Nutrition 2002; 21: 381-7.

7 Kassaye T, Receveur O, Johns T, Becklake MR. Prevalence of vitamin A deficiency in children aged 6-9 years in Wukro, northern Ethiopia. Bulletin of the World Health Organization 2001; 79: 415-22.

8 Chang Ying, Xian Zude. Child malnutrition in China present status and changing trend. Biomedical and Environmental Sciences 1999; 9: 164-80 (in Chinese).

9 Chen Mingxia, He Wu, Hu Zhengying, Fu Gang, Wang Yuying. Multiple factors analysis on malnutrition of children under 5 years in China in 2000. Journal of Hygiene Research 2003; 32: 249-53 (in Chinese).

$10 \mathrm{Hu} \mathrm{W}$, Tong S, Oldenburg B, Feng X. Serum vitamin A concentrations and growth in children and adolescents in Gansu Province, China. Asia Pacific Journal of Clinical Nutrition 2001; 10: 63-6.

11 National Statistic Bureau. Chinese Population in 1997. Beijing: China Statistics Publishing House, 1998 (in Chinese).

12 West KP Jr. Extent of vitamin A deficiency among preschool children and women of reproductive age. Journal of Nutrition 2002; 132: 2857S-66S.

13 Chinese Association of Pediatrics. The intervention protocol of subclinical vitamin A deficiency. Chinese Journal of Pediatrics 1995; 33: 201 (in Chinese).

14 Sloan NL, Rosen D, de la Paz T, Arita M, Temalilwa C, Solomons NW. Identifying areas with vitamin A deficiency: the validity of a semiquantitative food frequency method. American Journal of Public Health 1997; 87: 186-91.

15 Hu FB, Rimm E, Smith-Warner SA, Feskanich D, Stampfer MJ, Ascherio A, et al. Reproducibility and validity of dietary patterns assessed with a food-frequency questionnaire. American Journal of Clinical Nutrition 1999; 69: 243-9.

16 Clopper CJ, Pearson ES. The use of confidence or fiducial limits illustrated in the case of the binomial. Biometrika 1934; 26: 404-13.

17 Belsley DA, Welsch RE, Kuh E. Regression Diagnostics: Identifying Influential Data and Sources of Collinearity. New York: John Wiley \& Sons Inc., 1980.

18 Qin M, Ge CS, Dai JG. An investigation of subclinical vitamin A deficiency in 360 children 1-6 years of age. Chinese Journal of Pediatrics 1998; 36: 303-4 (in Chinese).

19 State Statistics Bureau of China. Classification of Chinese Health Status. Beijing: China Statistics Publishing House, 1994 (in Chinese).

20 Gorstein J, Shreshtra RK, Pandey S, Adhikari RK, Pradhan A. Current status of vitamin A deficiency and the national vitamin A control program in Nepal: results of the 1998 National Micronutrient Status Survey. Asia Pacific Journal of Clinical Nutrition 2003; 12: 96-103.

21 Ngare DK, Muttunga JN, Njonge E. Vitamin A deficiency in pre-school children in Kenya. East African Medical Journal 2000; 77: 421-4.

22 Ballew C, Bowman BA, Sowell AL, Gillespie C. Serum retinol distributions in residents of the United States: Third National Health and Nutrition Examination Survey, 1988-1994. American Journal of Clinical Nutrition 2001; 73: 586-93.

23 Underwood BA, Arthur P. The contribution of vitamin A to public health. FASEB Journal 1996; 10: 1040-8.

24 State Statistics Bureau of China. The Third Population Census of China: Results for Coastal, Inland and Western Region. Statistical Report. Beijing: Central Statistical Authority, 2002 (in Chinese).

25 Stephensen CB, Gildengorin G. Serum retinol, the acute phase response, and the apparent misclassification of vitamin A status in the third national health and nutrition examination survey. American Journal of Clinical Nutrition 2000; 72: 1170-8. 